\title{
Management of Obesity by Using CLA and Omega 3, 6,9 as Combined Therapy
}

\author{
Nidhi Sahu², Ayush Kumar ${ }^{3}$, Umama Yezdani ${ }^{1 *}$, Mohammad Gayoor khan ${ }^{2}$, Aarti Singh \\ Kushwah ${ }^{2}$, Priyanka Priya ${ }^{2}$, Shobha Kumari, Umesh Madhukar Patil, MD Habeeb Ali Mirza ${ }^{4}$, \\ Nazim \\ ${ }^{1}$ Department of Pharmacy Practice, MRM College of Pharmacy, Hyderabad, Telangana India \\ ${ }^{2}$ Department of Pharmacy, Truba Institute of Pharmacy, Bhopal, Madhya Pradesh India \\ ${ }^{3}$ Department of Pharmacy Practice, MMDU university, Mullana Ambala Haryana, India. \\ Department of nursing, Gautam Institute of Nursing and Pera medics ,Bihar India \\ Department of Pharmacy, A.R.A collage of pharmacy ,Dhule Maharashtra India \\ ${ }^{4}$ Department of Pharmacy Practice, St.Peters Institute of Pharmaceutical Sciences Warangal India \\ Department of Pharmaceutics, RGPV University Bhopal Madhya Pradesh, India
}

*Corresponding Author: Umama Yezdani, Department of Pharmacy Practice MRM College of Pharmacy Hyderabad.

\begin{abstract}
Obesity itself called Major disease in another term obesity is power house of the disease if the person is suffering obesity he/she might be near to very dangerous disease such as diabetes, high blood pressure, cholesterol, sickness, abnormal of hormone, low libido specially in male, low testosterone hormone production, blood clothing, heart pain, heart attack, vision problems etc. It is disorder which involving excessive body fat that increases the risk of health problems. The good news is that even modest weight loss can improve or prevent the health problems associated with obesity. Dietary changes, increased physical activity and behavior changes can help you lose weight. Prescription medications and weight-loss procedures are additional options for treating obesity.in this research article we are using omega 3,6,9 and CLA for the management of obesity and weight loss because weight loss is only way to decrease the risk level which outcomes in terms of obesity
\end{abstract}

Keywords: Obesity, CLA, Weight loss.

\section{INTRODUCTION}

Obesity is a complex disease involving an excessive amount of body fat. Obesity isn't just a cosmetic concern. It is a medical problem that increases your risk of other diseases and health problems, such as heart disease, diabetes, high blood pressure and certain cancers.There are many reasons why some people have difficulty avoiding obesity. Usually, obesity results from a combination of inherited factors, combined with the environment and personal diet and exercise choices. modest weight loss can improve or prevent the health problems associated with obesity. Dietary changes, increased physical activity and behavior changes can help you lose weight. Prescription medications and weight-loss procedures are additional options for treating obesity. Obesity is diagnosed when your body mass index (BMI) is 30 or higher. To determine your body mass index, divide your weight in pounds by your height in inches squared and multiply by 703. Or divide your weight in kilograms by your height in meters squared. For most people, BMI provides a reasonable estimate of body fat. However, BMI doesn't directly measure body fat, so some people, such as muscular athletes, may have a BMI in the obesity category even though they don't have excess body fat which is shown in figure 1.1 respectively. 


\begin{tabular}{ll}
\hline BMI & Weight status \\
\hline Below 18.5 & Underweight \\
\hline $18.5-24.9$ & Normal \\
\hline $25.0-29.9$ & Overweight \\
\hline 30.0 and higher & Obesity \\
\hline
\end{tabular}

Figure 1.1.

\section{CAUSeS}

Although there are genetic, behavioral, metabolic and hormonal influences on body weight, obesity occurs when you take in more calories than you burn through exercise and normal daily activities. Your body stores these excess calories as fat.

Most Americans and Indian diets are too high in calories - often from fast food and high-calorie beverages. People with obesity might eat more calories before feeling full, feel hungry sooner, or eat more due to stress or anxiety

\section{RISK FACTORS}

Obesity usually results from a combination of causes and contributing factors:

\subsection{Family Inheritance and Influences}

The genes you inherit from your parents may affect the amount of body fat store, and where that fat is distributed. Genetics may also play a role in how efficiently body converts food into energy, how body regulates appetite and how body burns calories during exercise. Obesity tends to run in families. That's not just because of the genes they share. Family members also tend to share similar eating and activity habits.

\subsection{Lifestyle Choices}

Unhealthy diet. A diet that's high in calories, lacking in fruits and vegetables, full of fast food, and laden with high-calorie beverages and oversized portions contributes to weight gain.

Liquid calories. People can drink many calories without feeling full, especially calories from alcohol. Other high-calorie beverages, such as sugared soft drinks, can contribute to significant weight gain. Inactivity. If you have a sedentary lifestyle, you can easily take in more calories every day than you burn through exercise and routine daily activities. Looking at computer, tablet and phone screens is a sedentary activity. The number of hours you spend in front of a screen is highly associated with weight gain.

\subsection{Certain Diseases and Medications}

In some people, obesity can be traced to a medical cause, such as Prader-Willi syndrome, Cushing syndrome and other conditions. Medical problems, such as arthritis, also can lead to decreased activity, which may result in weight gain.

Some medications can lead to weight gain if you don't compensate through diet or activity. These medications include some antidepressants, anti-seizure medications, diabetes medications, antipsychotic medications, steroids and beta blockers.

\subsection{Social and Economic Issues}

Social and economic factors are linked to obesity. Avoiding obesity is difficult if you don't have safe areas to walk or exercise. Similarly, may not have been taught healthy ways of cooking, or may not have access to healthier foods. In addition, the people spend time with may influence weight - more likely to develop obesity such as friends or relatives with obesity. 


\subsection{Age}

Obesity can occur at any age, even in young children. But as you age, hormonal changes and a less active lifestyle increase risk of obesity. In addition, the amount of muscle in body tends to decrease with age. Generally, lower muscle mass leads to a decrease in metabolism. These changes also reduce calorie needs, and can make it harder to keep off excess weight. If don't consciously control what eat and become more physically likely gain weight.

\subsection{Other Factors}

Pregnancy. Weight gain is common during pregnancy. Some women find this weight difficult to lose after the baby is born. This weight gain may contribute to the development of obesity in women. Breastfeeding may be the best option to lose the weight gained during pregnancy. Quitting smoking. Quitting smoking is often associated with weight gain. And for some, it can lead to enough weight gain to qualify as obesity. Often, this happens as people use food to cope with smoking withdrawal. In the long run, however, quitting smoking is still a greater benefit to your health than is continuing to smoke. doctor can help prevent weight gain after quitting smoking. Lack of sleep. Not getting enough sleep or getting too much sleep can cause changes.

\section{Material AND Methods}

- CLA: Conjugated linoleic acid (CLA) is a fatty acid found in meat and dairy that is believed to have various health benefits. It is also a popular weight loss supplement Linoleic acid is the most common omega- 6 fatty acid, found in large amounts in vegetable oils.

- Omega 3 - Souce includes [ fish and flaxseed, and in dietary supplements, such as fish oil.]

- ALA, DHA, DPA (Combined 3 fatty acid)

- omgea 6 - Souce includes [ soybeans.corn,safflower and sunflower oils,nuts and seeds,meat, poultry, fish and eggs.

- Omgea 9 - Souce includes [ olive oil, macadamia oil]

Method - Take 1 spoon Apple cider vinegar with luke warm water and combined polymeric capsule like omega 3,6,9 after meal and before dinner for 3 months' continuously.

\section{CONCLUSION}

In this article at last conclusion is condemned that after the three month's omega Cycle with apple cider vinegar it shows very great result in obesity person

\section{RESULT}

As noticed conclusion results are positively work with obesity and person suffering obesity he will managed this obesity by using these supplements

\section{ACKNOWLEDGEMENT}

First of all, I would like to thank Ms Umama Yezdani and others coauthor those help me for making this manuscript and support me time to time.

\section{REFERENCES}

[1] Mohammad Gayoor khan, Umama Yezdani,et.al.Gold Nanoparticle Sensor for the Detection of Ckd and Use of Nano-medicine in the Treatment of Kidney Diseases.ARC Journal of Nephrology. 2019;4(2):15-21.

[2] UmamaYezdani,Mohammad Gayoor khan, ZubiaZainab, Mayur sadar ,Shivam Choudghal, Damini Mishra, Hari Baska.The Current Scenario of Pharmaceutical Research; 3d Tissue Engineering Considered as Eminent Technique. ARC Journal of Public Health and Community Medicine.2019; 4(2):16-22.

[3] Gayoor Khan, Umama Yezdani, Rohit Verma, Raqshan Jabeen, Pradeep Sintha. Detection of Phlebovirus by using qualitative Real time (RT) - PCR and application of silver nanoparticles to control it. World J Pharm Pharm Sci.2018;7(11):936-52.

[4] Mohd.Gayoor Khan et.al.The Novel Drug Delivery System.World J Pharm Pharm Sci., 2017; 6(7):477-487. 
[5] Mohammad khan Gayoor, Yezdani Umama, Kumar ayush, sadar mayur, Rav Shorabh, Sahu Lalit, Use of Nanoparticles for the Treatment of Malignant Neoplasm As a Cancer Targeted Drug Delivery System. Asian Journal of Pharmaceutical Research and Development. 2019; 7(4):84-88, DOI: http://dx.doi.org/10.22270/ajprd.v7i4.513

[6] Mohd.Gayoor Khan.The Novel Drug Delivery System.World J Pharm Pharm Sci. 2017;6(7):477-487.

[7] Unama Yezdani, Mohd. Gayoor Khan, Fazal Khan, Arvind Verma, Nilesh Kushwah, Rohit Verma.The Drug Targeting in Alzheimer's or Applications \& it's Hazards. World J Pharm Sci.2017;7(11):1532-1549.

[8] Dr.H. S Chandel,Sharad P. Panday, Arvind Dangi, Ashish chaurasia, Mohd.Gayoor khan et al. Development of Targeted Drug delivery. International Journal of research methodology Ijrm.Human., 2017 Vol. 1 (2): 3034.

[9] Kushwah Nilesh, Yezdani Umama, Mohammad Gayoor khan, Manish kushwah, Kumar Ayush.The Fundamental of Novel Drug Delivery System; Methodology, Role of Nanotechnology; Nanoparticles in Pharmaceutical Research. International Journal of Emerging Technologies and Innovative Research (www.jetir.org), ISSN:2349-5162, Vol.6, Issue 6, page no.140-146, June-2019. DOI: http://doi.one/10.1729/Journal.21510

[10] Mohd. Gayoor Khan, Nilesh Kushwaha, Fazal Khan,Vipul Patel. Microencapsulation. International Journal of research methodology Ijrm.Human., 2017 Vol. 1 (2):35-42.

[11] Umama Yezdani, Mohd. Gayoor Khan, Nilesh Kushwah, Arvind Verma, Fazal Khan. Application of Nanotechnology in Diagnosis and treatment of various disease and it's future advances in medicine. World J Pharm Phar Sci.2018;7(11):1611-1633.

[12] Yezdani Umama, Venkatajah G, Rav Shourabh, Roshan Kumar, Arvind Verma, Ayush Kumar, Md. Khan Gayoor et al. The Scenario of pharmaceuticals and development of microwave assisted extraction technique. World J Pharm Pharm Sci.2019;8(7):1260-1271.

[13] Mohd.Gayoor khan et al, Radiopharmaceuticals Drug interactions. IJCRP,2017; Volume 1(5):40-47.

[14] Umama Yezdani1,Rohit verma,Ajay Kumar, Krishna Kumar Pandey, Mohd. Gayoor khan .The fundamental \& Development Role of epidural steroid injection in management of herniated intervertebral disc with Radiculopathy. IJCRP,2017; Volume 1(5):40-47.

[15] Mohammad Gayoor Khan, Yezdani Umama, Hari Baskar, Kumar Ayush, Karthikeyan Lakshman, Monika Sekar. Ethnopharmacological Studies of Argemone Mexicana for the Management of Psoriasis Followed By Molecular Techniques:Focus on Plant Metabolomics \& Mechanism of Action. International Journal of Basic Sciences and Applied Computing. 2019;2(8):1-5.DOI: 10.35940/ijbsac.H0097.072819

[16] Umama Yezdani,Mahmood Ali,Mohammad Gayoor Khan,Ayush Kumar, Prince Bhalla,Mayur Sadar. Pharmacological and Non Pharmacological approaches to vitiligo.World J Pharm Sci.2019;8(9):884-892.

[17] Gayoor KM, Kanta SN, Umama Y, Baskar H, Ayush K,Prasad TS. Ethnopharmacological Studies of Argemone mexicana for The Management of Psoriasis Followed by Molecular Techniques through Metabolomics. 2019;1(1): 1003.

[18] Mohammad Gayoor khan et al. The Novel drug delivery system. World Journal of Pharmacy and Pharmaceutical sciences,ISSN 2278 - 4357, 2017 Vol. 6 Issue (7): 477- 487.

[19] Mohammad Gayoor khan, et al, The Current Scenario of Pharmaceutical Research; 3d Tissue Engineering Considered as Eminent Technique.ARC Journal of Public Health and Community Medicine.2019; 4(2):1622. DOI: dx.doi.org/ 10.20431/2456-0596.0402003.

[20] Nagireddy, Syeda Majeeda Sultana, Syeda Mariya Anjum, Umama Yezdani, Kashifa Ayman et.al.Prevalence and causality Assessment of adverse drug reactions; clinical consequences of drug interactions and polypharmacy in patients presenting to secondary care hospital.Int. J. Of Res. In Pharmacology \& Pharmacotherapeutics. 2019;8(2):154-168.

[21] Mohammad Gayoor khan “A Systemic Review on Nanoparticles” SVOA Materials Science and Technology $1: 1(2019)$ 16-21.

[22] Damini Mishra, Mohammad Gayoor khan, Umama Yezdani , Shalini Jaswal, Vinesha Ravi,Shivam Choudghal, D Mukilan et.al. Systemic Review on Nanoparticles.SVOA Materials Science and Technology. 2019;1(1):16-21

[23] Dr. Umama Yezdani, et al. The fundamental \& Development role of epidural steroid injection in Management of herniated intervertebral disc with Radiculopathy. IJCRP. 2017; 1(5):40-47.

[24] Mohd Gayoor khan. Radiopharmaceuticals Drug interactions. IJCRP, 2017; 1(5):40-47.

[25] Umama Yezdani, Mohd. Gayoor Khan, Nilesh Kushwah, Arvind Verma,Fazal Khan. Application of Nanotechnology in Diagnosis and treatment of various disease and it's future advances in medicine. World J Pharm Pharm Sci.2018; 7(11):1611-1633. 
[26] Mohammad Gayoor Khan Umama Yezdani,Shivam Choudghal, Sana Rahman, Mayur Sadar, Ayush Kumar.A Brief Study on Motor Neuron Disease; and its Treatment Includes Drug Delivery System; Future Aspects on Neurological Science" IOSR Journal of Pharmacy and Biological Sciences (IOSR-JPBS) 14.4 (2019): 52-56.

[27] Mohammad Gayoor k, Umama Y, Shivam C, Mayur S, Rana S, et al., Gold Nanoparticle Sensor for The Detection of CKD and Use of Nanomedicine in The Treatment of Kidney Diseases. J Clin \& Commun Med 1(2)- 2020. JCCM.MS.ID.000109.

[28] Unama Yezdani,Mohd. Gayoor Khan, Fazal Khan, Arvind Verma, Nilesh Kushwah, Rohit Verma.THE DRUG TARGETING IN ALZHEIMER'S OR APPLICATIONS \& ITS HAZARDS. World J Pharm Pharm Sci.2018;7(11):1532-1549.

[29] Mohammad Gayoor Khan, Yezdani Umama, Hari Baskar, Kumar Ayush, Karthikeyan Lakshman, Monika Sekar. Ethnopharmacological Studies of Argemone Mexicana for the Management of Psoriasis Followed By Molecular Techniques: Focus on Plant Metabolomics \& Mechanism of Action International Journal of Basic Sciences and Applied Computing.2019;2(8):1-5. DOI: 10.35940/ijbsac.H0097.072819.

Citation: Umama Yezdani, et.al.," Management of Obesity by Using CLA and Omega 3, 6,9 as Combined Therapy.” ARC Journal of Pharmaceutical Sciences (AJPS), $6(1), \quad$ pp $\quad 1-5 . \quad$ DOI: http://dx.doi.org/10.20431/2455-1538.0601001

Copyright: (C) 2020 Authors. This is an open-access article distributed under the terms of the Creative Commons Attribution License, which permits unrestricted use, distribution, and reproduction in any medium, provided the original author and source are credited. 